\title{
2S and 3S State Masses and decay constants of heavy-flavour mesons in a non-relativistic QCD potential model with three-loop effects in $\mathrm{V}$-scheme
}

\author{
Rashidul Hoque $^{1,2, a}$, B. J. Hazarika ${ }^{1}$, D. K. Choudhury ${ }^{1,2}$ \\ ${ }^{1}$ Center of Theoretical Studies, Pandu College, Guwahati 781012, India \\ ${ }^{2}$ Department of Physics, Gauhati University, Guwahati 781 014, India
}

Received: 28 June 2020 / Accepted: 13 December 2020 / Published online: 30 December 2020

(C) The Author(s) 2020

\begin{abstract}
We make an analysis of three -loop effects of the strong coupling constant in the study of masses and decay constants of the heavy-flavour pseudo-scalar mesons (PSM) $D, D_{s}, \mathrm{~B}, B_{s}, B_{c}, \eta_{c}$ and $\eta_{b}$ in a non-relativistic QCD potential model using Dalgarno's perturbation theory (DPT). The first order mesonic wavefunction is obtained using Dalgarno's perturbation theory. The three-loop effects of strong coupling constant are included in the wave function in co-ordinate space and then used to examine the static and dynamic properties of the heavy-flavour mesons for $2 S$ and $3 S$ higher states. The results are compared with the other models available and are found to be compatible with available experimental values. In V-scheme, the three-loop effects on masses and decay constants of heavy-flavour mesons show a significant result.
\end{abstract}

\section{Introduction}

The non-relativistic predictions of Potential Models with a non-relativistic Hamiltonian for the heavy-light and heavyheavy mesons are found to be in fair agreement with the updated experimental data, theoretical results like QCD sum rule [1,2], Lattice results [3] and relativistic harmonic confinement model (RHCM) [53]. The static potential between the two heavy quarks is a fundamental quantity in QCD [4]. While its one loop corrections are computed in $[5,6]$, the corresponding two-loop effects were reported in late 1990's [7-9]. Numerical results are obtained first for fermionic contributions [10-12], whereas the analytical results are more recent [12]. Some important hadronic properties are the pseudoscalar meson mass $M_{P}$ and decay constant $f_{P}$. Phenomenological study of two-loop effects in the static and dynamic properties of heavy-flavour mesons using a linear cum Coulomb Cornell potential has been reported in the recent years $[14,15]$. In this work, quantum perturbation approach $[16,17]$ is used to calculate the approximate analytical forms of heavy flavored mesons. Here specifically the linear part of the potential $V(r)=-\frac{4 \alpha_{s}}{3 r}+b r$ is used as perturbation.

The most common perturbative method is Dalgarno's perturbation theory (DPT) [21,23-25], which is a stationary static perturbation theory. The non-relativistic potential model has been found successful for heavy-heavy $B, \eta_{c}$ and $\eta_{b}$ families. The study of the wave functions of heavy-flavour mesons like $\mathrm{B}$ and $\mathrm{D}$ and $\eta$ are important both analytically and numerically for studying the properties of strong interaction between heavy-light and heavy-heavy quarks as well as for investigating the mechanism of heavy meson decays. In this work, we have obtained a total first order corrected wavefunction for $2 S$ and $3 S$ states using Dalgarno's method of perturbation [30] with linear part of the Cornell potential $[19,31]$ as perturbation in co-ordinate space. This wavefunction is then used to estimate the masses and decay constants of heavy-light and heavy-heavy pseudo-scalar mesons in this improved QCD Potential model approach.

One aim of the present work is to make an analysis of the contribution of three-loop effects in the improved strong coupling constant $\alpha_{V}\left(\frac{1}{r}\right)$ in $V$-scheme, which in turn contributes to the spin-spin interaction [27-29] term present in the expression of mass and decay constant of the heavy-flavour meson. In addition, the non-relativistic binding energy effect between the two quark-antiquark composition of the heavyflavour meson is newly incorporated in the expression of PSM mass, which was absent in our some previous works [23-25,37,39,41].

\footnotetext{
a e-mail: rashidul999@gmail.com (corresponding author)
} 
The rest of the paper is organised as follows: Sect. 2 contains formalism, Sect. 3 contains results while Sect. 4 includes the conclusion.

\section{Formalism}

\subsection{V-scheme: three-loop effects}

$\mathrm{V}$-scheme is a standard way of taking into account the higher order effects of QCD, which are expressed as power series in the running strong coupling constant $\alpha \overline{M S}$ in $\overline{M S}$-scheme. The two-loop static potential in V-scheme which is also used as the three-loop static potential defined as $[8,33,34]$,

$V(r)=-\frac{C_{F} \alpha_{V}\left(\frac{1}{r}\right)}{r}$

Here, $\alpha_{V}$ is the effective strong coupling constant and $C_{F}$ is the color factor, given as, $C_{F}=\frac{\left(N_{C}^{2}-1\right)}{2 N_{C}}$, where $N_{C}$ is the no. of colors. Generally, the quark-gluon interaction is characterised by strong coupling constant $\alpha \overline{M S}\left(q^{2}\right)$ in a dimensionally independent $\overline{M S}$-scheme [7,20]. As discussed in the introduction, three-loop effects have been reported numerically first for fermionic contribution [10] followed by the gluonic counter part [10-12]. Analytic three-loop static potential have been discussed only in the year 2016 [12]. These effects are invariably reported in momentum space where the author sets $q^{2}=\mu^{\prime 2}$ as the renormalization scale to suppress the infrared logarithmics. For the three-loop effects, we follow [11] the numerical solution for the potential at three-loop level in momentum space is given by,

$$
\begin{aligned}
V(|q|)= & -\frac{4 \pi C_{F} \alpha_{s}\left(\overline{q^{2}} \mid\right)}{\overline{q^{2}}} \\
& \times\left[1+\left(\frac{\alpha_{s}}{\pi}\right)\left(2.5833-0.2778 n_{f}\right)\right. \\
& +\left(\frac{\alpha_{s}}{\pi}\right)^{2}\left(28.5468-4.1471 n_{f}+0.0772 n_{f}^{2}\right) \\
& +\left(\frac{\alpha_{s}}{\pi}\right)^{3}\left(209.884-51.4048 n_{f}\right. \\
& \left.\left.+2.9061 n_{f}^{2}-0.0214 n_{f}^{3}\right)\right]
\end{aligned}
$$

The corresponding expression in co-ordinate space will be,

$$
\begin{aligned}
V(r)= & -\frac{C_{F}}{r} \alpha_{s}\left(\mu^{\prime 2}\right)\left[1+\left(\frac{\alpha_{s}}{\pi}\right)\left(2.5833-0.2778 n_{f}\right)\right. \\
& +\left(\frac{\alpha_{s}}{\pi}\right)^{2}\left(28.5468-4.1471 n_{f}+0.0772 n_{f}^{2}\right)
\end{aligned}
$$

$$
\begin{aligned}
& +\left(\frac{\alpha_{s}}{\pi}\right)^{3}\left(209.884-51.4048 n_{f}\right. \\
& \left.\left.+2.9061 n_{f}^{2}-0.0214 n_{f}^{3}\right)\right]
\end{aligned}
$$

Using Eq. (1), we obtain the relationship between the improved strong coupling constant $\alpha_{V}\left(\frac{1}{r}\right)$ and the standard leading order strong coupling constant in $\overline{M S}$-scheme at $N^{3} L O$ level is given by:

$$
\begin{aligned}
\alpha_{V}\left(\frac{1}{r}\right)= & \alpha_{s}\left(\mu^{\prime 2}\right)\left[1+\left(\frac{\alpha_{s}}{\pi}\right)\left(2.5833-0.2778 n_{f}\right)\right. \\
& +\left(\frac{\alpha_{s}}{\pi}\right)^{2}\left(28.5468-4.1471 n_{f}+0.0772 n_{f}^{2}\right) \\
& +\left(\frac{\alpha_{s}}{\pi}\right)^{3}\left(209.884-51.4048 n_{f}\right. \\
& \left.\left.+2.9061 n_{f}^{2}-0.0214 n_{f}^{3}\right)\right]
\end{aligned}
$$

From the above equation, it is observed that at one-, twoand three-loop order, a large screening of the nonfermionic contributions by the $n_{f}$ terms which is most prominent in the case of $a_{3}$ for $n_{f}=5$.

\subsection{Dalgarno's perturbation theory}

\subsubsection{S state wave-function of the heavy-flavour mesons}

For shell L, we take $n=2$ and $l=0$; the $2 \mathrm{~S}$ state normalized wave-function [42] is given by:

$\psi_{20}^{(0)}(r)=\left(\frac{1}{2 \sqrt{2 \pi}}\right)\left(\frac{1}{a_{0}}\right)^{\frac{3}{2}}\left(1-\frac{r}{2 a_{0}}\right) e^{-\frac{r}{2 a_{0}}}$

In Dalgarno's perturbation theory, we make a small deformation to the Hamiltonian of the system as ,

$H=H_{0}+H^{\prime}$

where $H_{0}$ is the Hamiltonian of the unperturbed system and $H^{\prime}$ is the perturbed Hamiltonian. The approximation method is most suitable when $H$ is close to the unperturbed Hamiltonian $H_{0}$, i.e. $H^{\prime}$ is small. The standard potential is [19],

$V(r)=-\frac{4 \alpha_{V}}{3 r}+b r$.

This Coulomb-plus-linear potential, called Cornell potential is an important ingredient of the model which is established on the two kinds of asymptotic behaviours: ultraviolet at short distance (Coulomb like) and infrared at large distance (linear confinement term).

The Schrödinger equation takes the form as,

$$
H|\psi\rangle=\left(H_{0}+H^{\prime}\right)|\psi\rangle=E|\psi\rangle
$$

so that the first-order perturbed eigenfunction $\psi^{(1)}$ and eigen energy $W^{(1)}$ can be obtained using the relation,

$$
H_{0} \psi^{(1)}+H^{\prime} \psi^{(0)}=W^{(0)} \psi^{(1)}+W^{(1)} \psi^{(0)}
$$


where $H_{0}$ and $H^{\prime}$ are the parent and perturbed Hamiltonian defined as,

$$
\begin{aligned}
& H_{0}=\frac{-\nabla^{2}}{2 \mu}-\frac{A}{r} \\
& H^{\prime}=b r
\end{aligned}
$$

and

$$
\begin{aligned}
& W^{(0)}=\left\langle\psi^{(0)}\left|H_{0}\right| \psi^{(0)}\right\rangle=-\frac{\mu A^{2}}{2} \\
& W^{(1)}=\left\langle\psi^{(0)}\left|H^{\prime}\right| \psi^{(0)}\right\rangle
\end{aligned}
$$

With Cornell potential we get two choices in DPT:

1. Coulomb Parent, Linear Perturbation (CP).

2. Linear Parent, Coulomb Perturbation (LP).

In our present analysis we consider the first option. The radial Schrodinger equation for $l=0$ and $n=2$ is,

$$
\begin{aligned}
& {\left[-\frac{1}{2 \mu}\left(\frac{d^{2}}{d r^{2}}+\frac{2}{r} \frac{d}{d r}\right)+\frac{l(l+1)}{2}-\frac{A}{r}-W^{(0)}\right] \psi_{20}^{(1)}} \\
& =-\left[b r-W^{(1)}\right] \psi_{20}^{(0)} \\
& {\left[\frac{d^{2}}{d r^{2}}+\frac{2}{r} \frac{d}{d r}+\frac{2 \mu A}{r}-\mu^{2} A^{2}\right] \psi_{20}^{(1)}} \\
& =2 \mu\left[b r-W^{(1)}\right] \psi_{20}^{(0)}
\end{aligned}
$$

where $\psi_{20}^{(0)}$ is unperturbed wave function and is defined in Eq. (5) and $\psi_{20}^{(1)}$ is the first order correction to the wave function.

To solve the above equation, let us start from [24],

$\psi_{20}^{(1)}=\operatorname{br} R(r)$

With this substitution, Eq. (15) takes the form as:

$$
\begin{aligned}
& (b r) \frac{d^{2} R(r)}{d r^{2}}+4 b \frac{d R(r)}{d r}+\frac{2 b R(r)}{r}+\frac{2 b R(r)}{a_{0}}-\frac{b r R(r)}{a_{0}^{2}} \\
& =K\left(b r-W^{(1)}\right)\left(1-\frac{r}{2 a_{0}}\right) e^{-\frac{r}{2 a_{0}}}
\end{aligned}
$$

with,

$$
\begin{aligned}
K & =(2 \mu) \frac{1}{2 \sqrt{2 \pi}}\left(\frac{1}{a_{0}}\right)^{\frac{3}{2}} \\
a_{0} & =\frac{1}{\mu A} \\
A & =\frac{4 \alpha_{V}}{3}
\end{aligned}
$$

Putting as in [24],

$R(r)=F(r) e^{-\frac{r}{2 a_{0}}}$
Equation (17) becomes,

$$
\begin{aligned}
& (b r) F^{\prime \prime}(r)+\left(4 b-\frac{b r}{a_{0}}\right) F^{\prime}(r)+\left(\frac{2 b}{r}-\frac{3 b r}{4 a_{0}^{2}}\right) F(r) \\
& =K\left(b r-W^{(1)}\right)\left(1-\frac{r}{2 a_{0}}\right)
\end{aligned}
$$

The method of Frobenius [56] is a power series solution. Considering the only four terms in the series and neglecting the higher order terms for $2 S$ state,

$F(r)=\frac{A_{-1}}{r}+A_{0}+A_{1} r+A_{2} r^{2}$

with this the final form of the Eq. (22) is obtained as,

$$
\begin{aligned}
& \left(2 b a_{0}+\frac{b A_{-1}}{a_{0}}\right) \frac{1}{r}\left(6 b A_{1}-\frac{3 b A_{-1}}{4 a_{0}^{2}}\right) r^{0} \\
& +\left(12 b A_{2}-\frac{b A_{1}}{a_{0}}-\frac{3 b A_{0}}{4 a_{0}^{2}}\right) r-\left(\frac{2 b A_{2}}{a_{0}}+\frac{3 b A_{1}}{4 a_{0}^{2}}\right) r^{2} \\
& -\left(\frac{3 b A_{2}}{4 a_{0}^{2}}\right) r^{3}=-\left(K W^{1}\right) r^{0}+\left(K b+\frac{K W^{1}}{2 a_{0}}\right) r \\
& -\left(\frac{K b}{2 a_{0}}\right) r^{2}
\end{aligned}
$$

The expectation energy (eigen energy) is easily obtained using mathematica-9 as ,

$\left\langle W^{(1)}\right\rangle=\int_{0}^{\infty} 4 \pi r^{2} \psi^{0} H^{\prime} \psi^{0}=6 b a_{0}$

Equating co-efficients of $r^{-1}, r^{0} r$ and $r^{2}$ on both sides of Eq. (24), we get the values of constants as:

$$
\begin{aligned}
A_{-1} & =\frac{40 K a_{0}^{3}}{3} \\
A_{0} & =\frac{20 K a_{0}^{2}}{3} \\
A_{1} & =\frac{2 K a_{0}}{3} \\
A_{2} & =0
\end{aligned}
$$

Hence Eq. (16) becomes,

$\psi_{20}^{(1)}=K\left(K_{1}-K_{2} r+K_{3} r^{2}\right) e^{-\frac{r}{2 a_{0}}}$

with, $K_{1}=\frac{40 b a_{0}^{3}}{3}, K_{2}=\frac{20 b a_{0}^{2}}{3}, K_{3}=\frac{2 b a_{0}}{3}$.

Hence, using Dalgarno's perturbation theory with Coulombic parent, we get the total wave-function of the form corrected up to first order,

$$
\begin{aligned}
\psi_{20}^{\text {total }}(r) & =\psi_{20}^{(0)}(r)+\psi_{20}^{(1)}(r) \\
& =N\left[P-Q r+R r^{2}\right] e^{-\frac{r}{2 a_{0}}}, \\
P & =K\left[\frac{1}{2 \mu}+K_{1}\right]
\end{aligned}
$$




$$
\begin{aligned}
Q & =K\left[\frac{1}{4 \mu a_{0}}+K_{2}\right] \\
R & =K K_{3}
\end{aligned}
$$

where the normalization constant is,

$$
N=\frac{1}{\left[\int_{0}^{\infty} 4 \pi r^{2}\left[P-Q r+R r^{2}\right]^{2} e^{-\frac{r}{a_{0}}} d r\right]^{\frac{1}{2}}} .
$$

where, $a_{0}=\frac{1}{\mu \mathrm{A}}$; the value of $\mathrm{b}$ is $b=0.183 \mathrm{GeV}^{2}$ is the confinement parameter [5,9]. $\mu=\frac{m_{1} m_{2}}{m_{1}+m_{2}}$ is the reduced mass.

\subsubsection{S state wave-function of the heavy-flavour mesons}

For shell $\mathrm{M}$, we take $n=3$ and $l=0$; the $3 S$ state normalized wave-function [42] is given by,

$\psi_{30}^{(0)}(r)=\left(\frac{1}{3 \sqrt{3 \pi}}\right)\left(\frac{1}{a_{0}}\right)^{\frac{3}{2}}\left(1-\frac{2 r}{2 a_{0}}+\frac{2 r^{2}}{27 a_{0}^{3}}\right) e^{-\frac{r}{3 a_{0}}}$

represents the unperturbed wave function for $3 S$ state. For $l=0$ and $n=3$, the corresponding Eq. (15) for 3S state becomes,

$$
\begin{aligned}
& {\left[\frac{d^{2}}{d r^{2}}+\frac{2}{r} \frac{d}{d r}+\frac{2 \mu A}{r}-\mu^{2} A^{2}\right] \psi_{30}^{(1)}} \\
& =2 \mu\left[b r-W^{(1)}\right] \psi_{30}^{(0)}
\end{aligned}
$$

where $\psi_{30}^{(1)}$ is the first order correction to the wave function.

Similarly, as in Eq. (16) Let,

$\psi_{30}^{(1)}=\operatorname{br} R(r)$

with this substitution the Eq. (37) takes the form as,

$$
\begin{aligned}
& \text { (br) } \frac{d^{2} R(r)}{d r^{2}}+4 b \frac{d R(r)}{d r}+\frac{2 b R(r)}{r}+\frac{2 b R(r)}{a_{0}}-\frac{b r R(r)}{a_{0}^{2}} \\
& =L\left(b r-W^{(1)}\right)\left(1-\frac{2 r}{3 a_{0}}+\frac{2 r^{2}}{27 a_{0}^{3}}\right) e^{-\frac{r}{3 a_{0}}}
\end{aligned}
$$

with,

$L=(2 \mu) \frac{1}{3 \sqrt{3 \pi}}\left(\frac{1}{a_{0}}\right)^{\frac{3}{2}}$

The corresponding substitution as in Eq. (21);

$R(r)=F(r) e^{-\frac{r}{3 a_{0}}}$

Therefore, Eq. (39) is obtained as,

$(b r) F^{\prime \prime}(r)+\left(4 b-\frac{2 b r}{3 a_{0}}\right) F^{\prime}(r)+\left(\frac{2 b}{3 a_{0}}-\frac{8 b r}{9 a_{0}^{2}}+\frac{2 b}{r}\right) F(r)$

$$
=L\left(b r-W^{(1)}\right)\left(1-\frac{2 r}{3 a_{0}}+\frac{2 r^{2}}{27 a_{0}^{2}}\right)
$$

Similar way, the corresponding only four terms in the method of Frobenius [56] for $3 S$ state,

$F(r)=\frac{A_{-1}^{\prime}}{r}+A_{0}^{\prime}+A_{1}^{\prime} r+A_{2}^{\prime} r^{2}$

With this, the final form of the Eq. (42) is obtained as,

$$
\begin{aligned}
\left(2 b A_{0}^{\prime}+\frac{4 b A_{-1}^{\prime}}{3 a_{0}}\right) \frac{1}{r} & \\
+ & \left(6 b A_{1}^{\prime}+\frac{2 b A_{0}^{\prime}}{3 a_{0}}-\frac{8 b A_{-1}^{\prime}}{9 a_{0}^{2}}\right) r^{0} \\
+ & \left(12 b A_{2}^{\prime}-\frac{8 b A_{0}^{\prime}}{9 a_{0}^{2}}\right) r-\left(\frac{2 b A_{2}^{\prime}}{3 a_{0}}+\frac{8 b A_{1}^{\prime}}{9 a_{0}^{2}}\right) r^{2} \\
- & \left(\frac{8 b A_{2}^{\prime}}{9 a_{0}^{2}}\right) r^{3} \\
= & L\left[b r-\left(\frac{2 b}{3 a_{0}}\right) r^{2}+\left(\frac{2 b}{27 a_{0}^{2}}\right) r^{3}-W^{(1)}\right. \\
& \left.+\left(\frac{2 r}{3 a_{0}} W^{(1)}\right) r-\left(\frac{2 r^{2}}{27 a_{0}^{2}} W^{(1)}\right) r^{2}\right]
\end{aligned}
$$

The expectation energy is obtained using mathematica- 9 as ,

$\left\langle W^{(1)}\right\rangle=\frac{4 b}{a_{0}^{2}}$

Equating the co-efficients of $r^{-1}, r, r^{2}$ and $r^{3}$, we get the values of the constants as:

$$
\begin{aligned}
A_{-1}^{\prime} & =\frac{27 L a_{0}^{3}}{8}+\frac{9 L}{2} \\
A_{0}^{\prime} & =-\left(\frac{9 L a_{0}^{2}}{4}+\frac{3 L}{a_{0}}\right) \\
A_{1}^{\prime} & =\frac{39 L a_{0}}{48}+\frac{L}{3 a_{0}^{2}} \\
A_{2}^{\prime} & =-\frac{L}{12}
\end{aligned}
$$

Hence, Eq. (38) yields,

$\psi_{30}^{(1)}=b\left(A_{1}^{\prime}+A_{0}^{\prime} r+A_{1}^{\prime} r^{2}+A_{2}^{\prime} r^{3}\right) e^{-\frac{r}{3 a_{0}}}$

Therefore, the total wave-function corrected up to first order using Dalgarno's perturbation theory is,

$$
\begin{aligned}
\psi_{30}^{\text {total }}(r) & =\psi_{30}^{(0)}(r)+\psi_{30}^{(1)}(r) \\
& =N^{\prime}\left[L_{1}+L_{2} r+L_{3} r^{2}+L_{4} r^{3}\right] e^{-\frac{r}{3 a_{0}}}
\end{aligned}
$$


with,

$$
\begin{aligned}
K^{\prime} & =\frac{1}{3 \sqrt{3 \pi}}\left(\frac{1}{a_{0}}\right)^{\frac{3}{2}} \\
L_{1} & =\left[K^{\prime}+b A_{-1}^{\prime}\right] \\
L_{2} & =\left[b A_{0}^{\prime}-\frac{2 K^{\prime}}{3 a_{0}}\right] \\
L_{3} & =\left[b A_{1}^{\prime}+\frac{2 K^{\prime}}{27 a_{0}^{2}}\right] \\
L_{4} & =\left[b A_{2}^{\prime}\right]
\end{aligned}
$$

where the normalization constant is obtained from,

$$
N^{\prime}=\frac{1}{\left[\int_{0}^{\infty} 4 \pi r^{2}\left[L_{1}+L_{2} r+L_{3} r^{2}+L_{4} r^{3}\right]^{2} e^{-\frac{2 r}{3 a_{0}}} d r\right]^{\frac{1}{2}}} .
$$

\subsection{Wave function at origin (WFO)}

At origin, $\mathrm{r}=0$; WFO for $2 \mathrm{~S}$ state $\left(E q^{n} .31\right)$ is given by,

$\psi_{20}^{\text {total }}(0)=N P=N K\left(\frac{1}{2 \mu}+\frac{40 b a_{0}^{3}}{3}\right)$

Similarly, WFO, for $3 \mathrm{~S}$ state $\left(E q^{n} .51\right)$ is given by,

$\psi_{30}^{\text {total }}(0)=N^{\prime} L_{1}=N^{\prime}\left[K^{\prime}+b\left(\frac{27 L a_{0}^{3}}{8}+\frac{9 L}{2}\right)\right]$

\subsection{The expression of mass and decay constant of} pseudo-scalar meson

Fermi-Breit Hamiltonian: We take the non-relativistic two body Schrodinger equation (8) viz.,

$H|\psi\rangle=\left(H_{0}+H^{\prime}\right)|\psi\rangle=E|\psi\rangle$,

Where $H_{0}$ is the free Hamiltonian for two quarks of masses $m_{i}$ and $m_{j}$ and three momenta $P_{i}$ and $P_{j} . H_{0}$ is defined as,

$H_{0}=\frac{P_{i}^{2}}{2 m_{i}}+\frac{P_{j}^{2}}{2 m_{j}}$

and $H$ is the Fermi-Breit Hamiltonian with confinement which is defined as [51,55],

$H(r)=H^{\text {Conf }}(r)+H^{\text {hyp }}(r)+H^{\text {S.O. }}(r)$

Here,

$$
\begin{aligned}
H^{\text {Conf }}(r) & =\left(-\frac{\alpha_{s}(r)}{r}+\frac{3 b r}{4}+\frac{3 c}{4}\right)\left(\bar{F}_{i} \cdot \bar{F}_{j}\right) \\
H^{h y p}(r) & =-\frac{\alpha_{s}(r)}{m_{i} m_{j}}
\end{aligned}
$$

$$
\begin{aligned}
& \times\left[\frac{8 \pi}{3} \bar{S}_{i} \cdot \bar{S}_{j} \delta^{3}(r)+\frac{1}{r^{3}}\left(\frac{3\left(\bar{S}_{i} \cdot \bar{r}\right)\left(\bar{S}_{j} \cdot \bar{r}\right)}{r^{2}}-\bar{S}_{i} \cdot \bar{S}_{j}\right)\right]\left(\bar{F}_{i} \cdot \bar{F}_{j}\right) \\
H^{\text {S.O. }}(r)= & H^{\text {S.O. }(c . m .)}(r)+H^{\text {S.O. }(t . p)}(r) \\
H^{\text {S.o.(c.m.) }}(r)= & \frac{\alpha_{s}(r)}{r^{3}}\left(\frac{1}{m_{i}}+\frac{1}{m_{j}}\right)\left(\frac{S_{i}}{m_{i}}+\frac{S_{j}}{m_{j}}\right) L\left(\bar{F}_{i} \cdot \bar{F}_{j}\right) \\
H^{\text {S.O. (t.p) }}(r)= & -\frac{1}{2 r} \frac{\delta H^{\text {Conf }}}{\delta r}\left(\frac{S_{i}}{m_{i}^{2}}+\frac{S_{j}}{m_{j}^{2}}\right) L
\end{aligned}
$$

Here, $S_{i}$ and $S_{j}$ are the spins of the $i^{t h}$ and $j^{t h}$ quarks separated by a distance $r$. For ground state $(l=0)$, only the contact term proportional to $\delta^{3}(r)$ contributes and the Hamiltonian takes the simpler form as:

$H=\frac{4 \alpha_{s}}{3}\left(-\frac{1}{r}-\frac{8 \pi}{3} \delta^{3}(r) \frac{\bar{S}_{i} \cdot \bar{S}_{j}}{m_{i} m_{j}}\right)+b r+c$

In the present work, $c$ sets to be zero. To compute mass of the pseudoscalar mesons the spin-spin interaction possessing the form given by $[49,50]$,

$\left\langle H_{s s}\right\rangle=-\left.\frac{8 \pi \alpha_{s}}{3 m_{i} m_{j}} \psi(0)\right|^{2} \equiv-\frac{8 \pi \alpha_{s}}{3 m_{1} m_{2}}|\psi(0)|^{2}$

The mass and the decay constant of heavy-flavour pseudoscalar meson including only spin-spin interaction are given in $[26,27]$ as:

$M_{P}=m_{1}+m_{2}+E_{n, l}+\left\langle H_{s s}\right\rangle$

where, $m_{1}$ and $\mathrm{m}_{2}$ are the masses of quark-antiquark and $\alpha_{s}$ is the strong coupling constant identified as $\alpha_{V}$ in the present work, $E_{n, l}$ is the non-relativistic binding energy between the quark-antiquark composition and the van Royen-Weisskopf formula [54] for the decay of pseudo-scalar meson is,

$f_{P}=\sqrt{\frac{12|\psi(0)|^{2}}{M_{P}}}$

Here, $\alpha_{V}$ is the improved strong coupling constant in Vscheme defined by Eq. (4) and $|\psi(0)|$ is the wave function at origin (WFO).

The non-relativistic binding energy is given by [29,30,32],

$E_{n, l}=\frac{-2 \mu A^{2}}{(2(n-1)+1)^{2}+(2 l+1)^{2}+(4(n-1)+2)(2 l+1)}$

$\mathrm{S}$-waves states: For the $\mathrm{S}$-wave state $l=0$, we have;

$E_{n, l}=\frac{-2 \mu A^{2}}{(2(n-1)+1)^{2}+(4(n-1)+2)+1}$

Now, for $2 S$ state $(n=2)$ and for $3 S$ state $(n=3)$, the $E_{n, l}$ takes the form as:

$E_{2,0}=\frac{-2 \mu A^{2}}{16}$ 
and

$E_{3,0}=\frac{-2 \mu A^{2}}{36}$

\section{Results}

\subsection{Input parameters used in the calculation}

With the formalism developed in Sec.2, we calculate the masses and decay constants of pseudo-scalar heavy-flavour mesons using Eqs. (70) and (71) in $2 S$ and $3 S$ states, which are shown in Tables $6,7,8$ and 9 respectively. The input parameters are taken as in $[24,36,37,52] ; m_{u / d}=$ $0.336 \mathrm{GeV}, m_{b}=4.95 \mathrm{GeV}, m_{c}=1.55 \mathrm{GeV}, m_{s}=$ $0.483 \mathrm{GeV}$, and $b=0.183 \mathrm{GeV}^{2}$. Also we calculated the effective strong coupling constant in V-scheme and found $\alpha_{V}\left(\frac{1}{r}\right)=0.73$ for $n_{f}=4$ and $\alpha_{V}\left(\frac{1}{r}\right)=0.303$ for $n_{f}=4$ at $N^{3} L O$ level respectively. We make a comprehensive comparison of our results with QCD sum rule [1,2], Lattice results $[3,19,43,44]$ other models like [24], RHCM [53] and the recent experimental values [36].

We use the usual expression for strong coupling constant in $\overline{M S}$ scheme for lowest order (LO level) is given by [40]

$\alpha_{S}\left(q^{2}\right)=\alpha \overline{M S}\left(q^{2}\right)=\frac{4 \pi}{\beta_{0} \ln \left(\frac{m_{Q}^{2}}{\Lambda_{Q C D}^{2}}\right)}$

Here, $m_{Q}$ is the mass of the heavy quark and $\Lambda_{Q C D}$ is the QCD scale parameter having values $0.292 \mathrm{GeV}$ and $0.210 \mathrm{GeV}$ for $n_{f}=4$ and $n_{f}=5$ respectively.

$3.2 \alpha_{V}, E_{n, l}$ and different parameters used in V-scheme

\subsubsection{Calculation of effective strong coupling constant $\alpha_{V}\left(\frac{1}{r}\right)$}

Using Eq. (4), we tabulate the values of $\alpha_{V}\left(\frac{1}{r}\right)$ taking into account one-loop (NLO), two-loop $\left(N^{2} L O\right)$ and three-loop $\left(N^{3} L O\right)$ in Table 1 for $n_{f}=4$ and $n_{f}=5$. It shows that for $n_{f}=4$, the enhancements are respectively $21 \%, 48 \%$ and $62 \%$ while for $n_{f}=5$, the corresponding enhancements are $8 \%, 14 \%$ and $17 \%$ respectively. The anti-screening effects of gluons seem to play an important role for $n_{f}=5$.

Table 1 Values of $\alpha_{V}\left(\frac{1}{r}\right)$

\begin{tabular}{lllll}
\hline$n_{f}$ & $L O$ & $N L O$ & $N^{2} L O$ & $N^{3} L O$ \\
\hline 4 & 0.45 & 0.5445 & 0.67 & 0.73 \\
5 & 0.259 & 0.28 & 0.297 & 0.303 \\
\hline
\end{tabular}

\subsubsection{Calculation of non-relativistic binding energy $E_{n, l}$}

Following Eqs. (74) and (75) along with Eq. (20) we obtain the non-relativistic binding energy $E_{n, l}$ (in $\mathrm{GeV}$ ) of the heavy-flavour pseudo-scalar mesons $D, D_{s}, B, B_{s}, B_{c}, \eta_{c}$ and $\eta_{b}$ respectively and tabulated them in Table 2 below.

It is seen that from the above Table 2, the magnitude of the non-relativistic binding energy is always greater for $2 S$ state than $3 S$ state, which indicates, the non-relativistic binding energy decreases with increasing higher states.

\subsubsection{Calculation of parameter $A$}

Using Eq. (20) with the values of $\alpha_{V}$ from Table 1, we calculate the parameter $A$ for the same heavy-flavour pseudoscalar mesons given in the Table 3 below.

\subsubsection{Calculation of different parameters used in the expression of mass $M_{P}$}

For the mentioned seven heavy-flavour pseudo-scalar mesons $D, D_{s}, B, B_{s}, B_{c}, \eta_{c}$ and $\eta_{b}$ respectively, the Eqs. (18), (19), (20), (32), (33), (34) and (35) along with Eqs. (58), (69) and (70) yield the values of the following parameters (Table 4):

Similarly, for the same heavy-flavour pseudo-scalar mesons, the corresponding Eqs. (19), (20), (52), (53), (54), (55), (56), (57) along with Eqs. (59), (69) and (70) yield the following Table 5.

Table 2 Values $E_{n, l}$ in $\mathrm{GeV}$ for $2 S$ and $3 S$ states

\begin{tabular}{lllll}
\hline Meson & State & $\mathrm{E}_{20}$ & State & $\mathrm{E}_{30}$ \\
\hline$D(c \bar{u} / c \bar{d})$ & $2 \mathrm{~S}$ & -0.03 & $3 \mathrm{~S}$ & -0.0145 \\
$D_{s}(c \bar{s})$ & $2 \mathrm{~S}$ & -0.0432 & $3 \mathrm{~S}$ & -0.0194 \\
$B(u \bar{b} / d \bar{b})$ & $2 \mathrm{~S}$ & -0.0064 & $3 \mathrm{~S}$ & -0.00285 \\
$B_{S}(s \bar{b})$ & $2 \mathrm{~S}$ & -0.009 & $3 \mathrm{~S}$ & -0.004 \\
$B_{c}(\bar{b} c)$ & $2 \mathrm{~S}$ & -0.0241 & $3 \mathrm{~S}$ & -0.011 \\
$\eta_{c}(c \bar{c})$ & $2 \mathrm{~S}$ & -0.0158 & $3 \mathrm{~S}$ & -0.00703 \\
$\eta_{b}(b \bar{b})$ & $2 \mathrm{~S}$ & -0.051 & $3 \mathrm{~S}$ & -0.002245
\end{tabular}

Table 3 Values of parameter $A$ at $L O, N L O, N^{2} L O$ and $N^{3} L O$ level

\begin{tabular}{lllll}
\hline Meson & $A(L O)$ & $A(N L O)$ & $A\left(N^{2} L O\right)$ & $A\left(N^{3} L O\right)$ \\
\hline$D(c \bar{u} / c \bar{d})$ & 0.53 & 0.72 & 0.89 & 0.97 \\
$D_{s}(c \bar{s})$ & 0.53 & 0.72 & 0.89 & 0.97 \\
$B(u \bar{b} / d \bar{b})$ & 0.345 & 0.373 & 0.396 & 0.404 \\
$B_{S}(s \bar{b})$ & 0.345 & 0.373 & 0.396 & 0.404 \\
$B_{c}(\bar{b} c)$ & 0.345 & 0.373 & 0.396 & 0.404 \\
$\eta_{c}(b \bar{c})$ & 0.345 & 0.373 & 0.396 & 0.404 \\
$\eta_{b}(b \bar{b})$ & 0.345 & 0.373 & 0.396 & 0.404
\end{tabular}


Table 4 Values of parameters used and $|\psi(0)|^{2}$ (in $\mathrm{GeV}^{3}$ ) in $2 S$ State

\begin{tabular}{llllllll}
\hline Meson & $\mathrm{a}_{0}$ & $\mathrm{~K}$ & $\mathrm{P}$ & $\mathrm{Q}$ & $\mathrm{R}$ & $\mathrm{N}$ & $|\psi(0)|^{2}$ \\
\hline$D(c \bar{u} / c \bar{d})$ & 3.72 & 0.01529 & 1.948 & 0.3 & 0.007 & 0.042 & 0.0066 \\
$D(c \bar{s})$ & 2.80 & 0.03134 & 1.72 & 0.307 & 0.017 & 0.055 & 0.00895 \\
$B(u \bar{b} / d \bar{b})$ & 7.88 & 0.00566 & 5.98 & 0.45 & 0.006 & 0.0083 & 0.0025 \\
$B_{s}(s \bar{b})$ & 5.62 & 0.0132 & 5.73 & 0.510 & 0.0091 & 0.0096 & 0.003 \\
$B_{c}(\bar{b} c)$ & 2.10 & 0.15498 & 3.556 & 0.8495 & 0.0404 & 0.064 & 0.052 \\
$\eta_{c}(c \bar{c})$ & 1.3256 & 0.2026 & 1.282 & 0.4836 & 0.033 & 0.32 & 0.168 \\
$\eta_{b}(b \bar{b})$ & 1.0001 & 0.98745 & 2.61 & 1.304 & 0.1205 & 0.2425 & 0.401 \\
\hline
\end{tabular}

Table 5 Values of parameters used and $|\psi(0)|^{2}$ (in $\mathrm{GeV}^{3}$ ) in 3S state

\begin{tabular}{llllllllll}
\hline Meson & $\mathrm{a}_{0}$ & $\mathrm{~K}^{\prime}$ & $\mathrm{L}$ & $\mathrm{L}_{1}$ & $\mathrm{~L}_{2}$ & $\mathrm{~L}_{3}$ & $\mathrm{~L}_{4}$ & $\mathrm{~N}^{\prime}$ & $|\psi(0)|^{2}$ \\
\hline$D(c \bar{u} / c \bar{d})$ & 3.72 & 0.01514 & 0.008358 & 0.289 & 0.08 & 0.0053 & 0.00009 & 0.114 & 0.0011 \\
$D_{s}(c \bar{s})$ & 2.80 & 0.02318 & 0.01706 & 0.2685 & 0.079 & 0.0066 & 0.00014 & 0.14 & 0.00165 \\
$B(u \bar{b} / d \bar{b})$ & 7.88 & 0.0049 & 0.00308 & 0.94 & 0.088 & 0.0032 & 0.00004 & 0.053 & 0.00249 \\
$B_{s}(s \bar{b})$ & 5.62 & 0.00815 & 0.007174 & 0.803 & 0.12 & 0.0064 & 0.0001 & 0.064 & 0.00262 \\
$B_{c}(\bar{b} c)$ & 2.10 & 0.035688 & 0.08422 & 0.59 & 0.2 & 0.028 & 0.001 & 0.1032 & 0.0038 \\
$\eta_{c}(c \bar{c})$ & 1.3256 & 0.071159 & 0.110297 & 0.854 & 0.180 & 0.0289 & 0.0014 & 0.12 & 0.0105 \\
$\eta_{b}(b \bar{b})$ & 1.0001 & 0.108589 & 0.5375 & 0.89 & 0.59 & 0.125 & 0.007 & 0.19 & 0.030 \\
\hline
\end{tabular}

3.3 $2 \mathrm{~S}$ State masses and decay constants of heavy-flavour mesons and their comparison

Following Eqs. (58), (70), (71) and (76) along with the results of Tables 1, 2, 3 and 4; we calculate the masses $M_{P}$ and decay constants $f_{P}$ (in $\mathrm{GeV}$ ) of the heavy-flavour pseudo-scalar mesons $D, D_{s}, B, B_{s}, B_{c}, \eta_{c}$ and $\eta_{b}$ and tabulated them in Tables 6 and 7 respectively with the comparison of results of the other models like QCD sum rule [1,2], Lattice results $[3,19,43,44]$, relativistic harmonic potential model (RHPM) [53] and experimental data [36,45-47].

\subsection{S State masses and decay constants of heavy-flavour mesons and their comparison}

Using Eqs. (59), (70), (71) and (76) along with the results of Tables 1, 2, 3 and 5; we calculate the masses $M_{P}$ and decay constants $f_{P}$ (in $\mathrm{GeV}$ ) of the same heavy-flavour pseudoscalar mesons and tabulated them in Tables 8 and 9.

Comparative analysis of pseudo-scalar meson mass spectra from Tables 6 and 8 provides a well agreement of our results in both $2 S$ and $3 S$ states while the decay constants from Tables 7 and 9 are found to be in better harmony only for $2 S$ state with experimental values and other models available.
3.5 Sources of uncertainties and possible future improvements

Let us discuss the sources of uncertainties in the calculation and the possible future improvements. The sources of uncertainties arise mainly:

(a) Due to the numerical values of quark masses. But for heavy-light meson, the uncertainty in the light quark mass does not play any significant role for $m_{Q} \gg m_{q}$. i.e., when the heavy quark mass is very large compared to the light quark mass.

(b) Another uncertainty is in the definition of strong coupling constant in the $\overline{M S}$-scheme for lowest order (LO level) defined by Eq. (76). Here the possible uncertainty is in preferring the choice of scale $Q^{2}=m_{Q}^{2}$, where $m_{Q}^{2}$ is the square of the mass of heavy quark. However, even in an alternative choice of the scale $Q^{2}=m_{\text {meson }}^{2}$, the differences in the predictions are still insignificant for heavy-light mesons as $m_{Q} \gg m_{q}$. For heavy-heavy mesons like $\eta_{c}$ and $\eta_{b}$, where both the quarks are heavy, there will be a very minor change in the predictions of mass spectra and decay constants. 
Table 6 Mass $\left(M_{P}\right)$ in $\mathrm{GeV}$

\begin{tabular}{lllllll}
\hline Meson & $\mathrm{M}_{\mathrm{P}(\mathrm{Our})}$ & RHCM [53] & Ref. [24] & Lattice [3] & Q. sum rule [1,2] & Exp. M [36,45-47] \\
\hline$D(c \bar{u} / c \bar{d})$ & 1.80 & 2.653 & 2.378 & 1.885 & 1.87 & $1.869 \pm 0.0016$ \\
$D_{s}(c \bar{s})$ & 1.886 & 2.778 & 2.500 & 1.969 & 1.97 & $1.968 \pm 0.0033$ \\
$B(u \bar{b} / d \bar{b})$ & 5.277 & 6.192 & 5.798 & 5.283 & 5.28 & $5.279 \pm 0.0017$ \\
$B_{s}(s \bar{b})$ & 5.420 & 6.264 & 5.902 & 5.366 & 5.37 & $5.366 \pm 0.0024$ \\
$B_{c}(\bar{b} c)$ & 6.45 & 6.929 & 6.810 & 6.278 & $6.277 \pm 0.006$ \\
$\eta_{c}(c \bar{c})$ & 2.91 & 3.626 & & & $2.980 \pm 0.0012$ \\
$\eta_{b}(b \bar{b})$ & 9.80 & 10.012 & & & $9.461 \pm 0.0026$ \\
\hline
\end{tabular}

Table 7 Decay constant $\left(f_{P}\right)$ in $\mathrm{GeV}$

\begin{tabular}{llllll}
\hline Meson & $\mathrm{f}_{\mathrm{P}(\text { Our })}$ & RHCM [53] & QCD sum [1,2] & Latt. [3,19,43,44] & Exp. f \\
\hline$D(c \bar{u} / c \bar{d})$ & 0.209 & 0.336 & $0.206 \pm 0.002$ & $0.220 \pm 0.003$ & $0.205 \pm 0.085 \pm 0.025^{[45,46]}$ \\
$D_{s}(c \bar{s})$ & 0.238 & 0.387 & $0.245 \pm 0.015$ & $0.258 \pm 0.001$ & $0.254 \pm 0.059^{[45,46]}$ \\
$B(u \bar{b} / d \bar{b})$ & 0.100 & 0.581 & $0.193 \pm 0.012$ & $0.218 \pm 0.005$ & $0.198 \pm 0.014^{[47]}$ \\
$B_{s}(s \bar{b})$ & 0.107 & 0.600 & $0.232 \pm 0.018$ & $0.228 \pm 0.010$ & $0.237 \pm 0.017^{[47]}$ \\
$B_{c}(\bar{b} c)$ & 0.311 & 0.607 & & & $0.562^{[48]}$ \\
$\eta_{c}(c \bar{c})$ & 0.832 & 0.420 & & & \\
$\eta_{b}(b \bar{b})$ & 0.699 & 0.711 & & & \\
\hline
\end{tabular}

Table 8 Mass $\left(M_{P}\right)$ in $\mathrm{GeV}$

\begin{tabular}{lllllll}
\hline Meson & $\mathrm{M}_{\mathrm{P}(\text { Our })}$ & RHCM [53] & Ref. [24] & Lattice [3] & Q.sum rule [1,2] & Exp. MP [36,45-47] \\
\hline$D(c \bar{u} / c \bar{d})$ & 1.860 & 3.162 & 2.378 & 1.885 & 1.87 & $1.869 \pm 0.0016$ \\
$D(c \bar{s})$ & 1.997 & 3.264 & 2.500 & 1.969 & 1.97 & $1.968 \pm 0.0033$ \\
$B(u \bar{b} / d \bar{b})$ & 5.279 & 6.732 & 5.798 & 5.283 & 5.28 & $5.279 \pm 0.0017$ \\
$B_{s}(s \bar{b})$ & 5.425 & 6.764 & 5.902 & 5.366 & 5.37 & $5.366 \pm 0.0024$ \\
$B_{c}(\bar{b} c)$ & 6.48 & 7.308 & 6.810 & 6.278 & $6.277 \pm 0.006$ \\
$\eta_{c}(c \bar{c})$ & 3.08 & 4.047 & & & $2.980 \pm 0.0012$ \\
$\eta_{b}(b \bar{b})$ & 9.87 & 10.319 & & & $9.461 \pm 0.0026$ \\
\hline
\end{tabular}

Table 9 Decay constant $\left(f_{P}\right)$ in $\mathrm{GeV}$

\begin{tabular}{llllll}
\hline Meson & $\mathrm{f}_{\mathrm{P}(\text { Our })}$ & RHCM [53] & QCD sum [1,2] & Latt. [3,19,43,44] & Exp. f \\
\hline$D(c \bar{u} / c \bar{d})$ & 0.100 & 0.336 & $0.206 \pm 0.002$ & $0.220 \pm 0.003$ & $0.205 \pm 0.085 \pm 0.025^{[45,46]}$ \\
$D_{s}(c \bar{s})$ & 0.110 & 0.387 & $0.245 \pm 0.015$ & $0.258 \pm 0.001$ & $0.254 \pm 0.059^{[45,46]}$ \\
$B(u \bar{b} / d \bar{b})$ & 0.085 & 0.581 & $0.193 \pm 0.012$ & $0.218 \pm 0.005$ & $0.198 \pm 0.014^{[47]}$ \\
$B_{s}(s \bar{b})$ & 0.087 & 0.600 & $0.232 \pm 0.018$ & $0.228 \pm 0.010$ & $0.237 \pm 0.017^{[47]}$ \\
$B_{c}(\bar{b} c)$ & 0.09 & 0.607 & & & $0.562^{[48]}$ \\
$\eta_{c}(c \bar{c})$ & 0.205 & 0.420 & & & \\
$\eta_{b}(b \bar{b})$ & 0.200 & 0.711 & & & \\
\hline
\end{tabular}


Let us now discuss about the possible future improvements and their applications: In the present work we have not introduced the relativistic effect in the light quark. For the future improvement the light quark can be considered relativistically with the Hamiltonian $H=M+\frac{p^{2}}{2 m}+\sqrt{p^{2}+m^{2}}+V(r)$, where $\mathrm{M}$ is the mass of heavy quark and $\mathrm{m}$ is the mass of light quark as in $[33,37,57]$. Similarly, the QCD correction factor $\bar{C}^{2}\left(\alpha_{V}\right)[37,58]$ can also be introduced in van RoyenWeisskopf formula [37,54] for the decay of pseudo-scalar meson. The improved model then can be applied in the study of Branching ratio, Oscillation frequency, Leptonic decay as well [37].

\section{Conclusion}

The spectroscopic properties of heavy-light and extended heavy-heavy favour system mesons are studied as a potential scheme with Dalgarno's Perturbation Theory. The study of mass spectroscopy and decay properties of the heavy-flavour mesonic states considering only spin-spin interaction provides us a fruitful results of the dynamics of quarks and gluons at the hadronic scale. The values of masses are found to be $1.80,1.886,5.277,5.420,6.45,2.91$ and $9.80 \mathrm{GeV}$ and decay constants are found to be $0.209,0.238,0.100,0.107$, $0.311,0.832$, and $0.699 \mathrm{GeV}$ for $D, D_{s}, B, B_{s}, B_{c}, \eta_{c}$ and $\eta_{b}$ respectively for pseudo-scalar mesons in $2 S$ state. The corresponding masses and decay constants of the above seven pseudo-scalar mesons for $3 S$ state are found to be 1.860 , 1.997, 5.279, 5.425, 6.48, 3.08, 9.87 GeV and 0.100, 0.110, $0.085,0.087,0.09,0.205,0.200 \mathrm{GeV}$ respectively. The values of $M_{P}$ and $f_{P}$ are in close agreement with the values available from other models and experimental data for $2 S$ state. For $3 S$ state the $M_{P}$ values are in close proximity with other models but some of $f_{P}$ values are smaller than the other models and experimental data. The success suggests the importance of the effects of three-loop contribution and the non-relativistic binding energy between the quark composite and the choice of renormalization scale parameter used as well as the potential forms for the understanding of the dynamics of the light-heavy and heavy-heavy quark systems. However, although mass spectroscopic results are quite good for $3 S$ state, the values of decay constants $f_{P}$ are smaller due to the small WFO values. It suggests limitation of the van Royen-Weisskopf formula $\left(E q^{n} .71\right)$. Proper incorporation of QCD corrections $\bar{C}^{2}\left(\alpha_{V}\right)[37,58]$ and relativistic effects at least minimally [37] are expected to improve the results.

In conclusion, the Dalgarno's Perturbation approach with the option of linear perturbation and three-loop effects employed here is found to be successful in the study of heavyflavour mesons with a Coulombic plus linear potential. More- over, our results suggest, the treatment of light-flavour relativistically and heavy-flavour non-relativistically seems to be appropriate in light of the successful predictions of the various properties of heavy-light and heavy-heavy systems. The parameters and the results obtained here can be useful in the study of the leptonic and semi-leptonic decays of these mesons.

Acknowledgements One of the authors (R. Hoque) acknowledges Maulana Azad National Fellowship, India for financial support by providing Fellowship during the research work. He also thanks the Head of the department of Physics, Gauhati University and the Head of the department of Physics, Pandu College, Guwahati, India for providing the necessary facilities.

Data Availability Statement This manuscript has no associated data or the data will not be deposited. [Authors' comment: Our research work is basically to build a model to estimate static and dynamic properties of heavy-flavour mesons within phenomenological Quantum Chromodynamics. So, data deposition is not necessarily emphasized in our present work.]

Open Access This article is licensed under a Creative Commons Attribution 4.0 International License, which permits use, sharing, adaptation, distribution and reproduction in any medium or format, as long as you give appropriate credit to the original author(s) and the source, provide a link to the Creative Commons licence, and indicate if changes were made. The images or other third party material in this article are included in the article's Creative Commons licence, unless indicated otherwise in a credit line to the material. If material is not included in the article's Creative Commons licence and your intended use is not permitted by statutory regulation or exceeds the permitted use, you will need to obtain permission directly from the copyright holder. To view a copy of this licence, visit http://creativecomm ons.org/licenses/by/4.0/.

Funded by SCOAP ${ }^{3}$.

\section{References}

1. W. Lucha et al., J. Phys. G Nucl. Part. Phys. 38, 105002 (2011)

2. Z.G. Wang, Eur. Phys. J. C 75, 427 (2015). https://doi.org/10.1140/ epjc/s10052-015-3653-9

3. R.J. Dowdall et al., HPQCD Collab., arXiv:1207.5749v1

4. N. Brambilla et al., Quarkonium Working Group, arXiv:hep-ph/0412158

5. W. Fischler, Nucl. Phys. B 129, 157 (1977)

6. A. Billoire, Phys. Lett. B 92, 343 (1980)

7. M. Peter, Phys. Rev. Lett. 78, 602 (1997). arXiv:hep-ph/9610209

8. M. Peter, Phys. B 501 (1997). arXiv:hep-ph/9702245

9. Y. Schroder, The static potential in QCD to two loops, DESY-98191. arXiv:hep-ph/9812205

10. Alexander V. Smirnov et al., Fermionic contributions to the threeloop static potential. PLB 668, 293 (2008). arXiv:0809.1927v1

11. Alexander V. Smirnov et al., Three-loop static potential. PRL 104, 112002 (2010). arXiv:0911.4742v2

12. Roman N. Lee et al., Phys. Dev. D 94, 054029 (2016)

13. C. Anzai, Y. Kiyo, Y. Sumino, PRL 104, 112003 (2010)

14. M. Melles, Static QCD potential in co-ordinate space with quark masses through two-loops. Phys. Rev. D 62, 074019 (2000)

15. D.K. Choudhury, N.S. Bordoloi, Mod. Phys. Lett. A. 24(6), 443451 (2009)

16. K.K. Pathak, D.K. Choudhury, Chin. Phys. Lett. 28, 101201 (2011) 
17. D.K. Choudhury, N.S. Bordoloi, Mod. Phys. Lett. A. 17(29), 19091913 (2002)

18. Y. Schroder, The static potential in QCD to two loops, DESY-98191. arXiv:hep-ph/9812205

19. E. Eichten et al., Phys. Rev. D 21, 203 (1980)

20. S.J. Brodsky, Commensurate scale relations and the Abelian correspondence principle, SLAC-PUB-7861 (1998)

21. A. Dalgarno, Stationary perturbation theory, in Quantum Theory: I Elements, ed. by D.R. Bates (Academic Press, New York, 1961)

22. D.K. Choudhury, N.S. Bordoloi, Int. J. Mod. Phys. A 15(23), 36673678 (2000)

23. K.K. Pathak, D.K. Choudhury, N.S. Bordoloi, Leptonic decay of heavy-light mesons in a QCD potential model. Int. J. Mod. Phys. A (2012). https://doi.org/10.1142/S0217751X13500103

24. T. Das et al., Int. J Mod. Phys. A (2016). https://doi.org/10.1142/ S0217751X1650189X

25. T. Das, D.K. Choudhury, K.K. Pathak, Indian J. Phys. 90, 13071312 (2016). https://doi.org/10.1007/S12648-016-0866-1

26. W. Lucha, F.F. Schoberl, D. Gromes, Bound states of quarks. Phys. Rep. (Rev. Sect. Phys. Lett.) 200, 127-240 (1991)

27. H. Hassanabadi, M. Ghafourian, S. Rahmani, Study of heavylight mesons properties via the variational method for Cornell potential interaction. Few-Body Syst. https://doi.org/10.1007/ s00601-015-1040-6

28. C. Quigg, J.L. Rosner, Phys. Rep. 56, 167 (1979)

29. M. Moazami, H. Hassanabadi, S. Zarrinkamar, Heavy-light mesons under a new potential containing Cornell, Gaussian and inverse square terms. Few Body Syst. https://doi.org/10.1007/ s00601-018-1422-7

30. A. Ghataak, S. Lokanathan, Quantum Mechanics, Theory and Applications (TRINITY, New Delhi, 2017)

31. D. Griffiths, Introduction to Elementary Particles (Wiley, New York, 1987), p. 158

32. F. Halzen, A.D., Martin, Quarks and Leptons. Wiley, New York. ISBN:0-471-88741-2

33. A.K. Rai, R.H. Parmar, P.C. Vinodkumar, J. Phys. G Nucl. Part. Phys. 28, 22752282 (2002)

34. J. Lahkar, D.K. Choudhury, B.J. Hazarika, arXiv:1902.02079v1

35. G.P. Lepage, P.B. Mackenzie, Phys. Rev. D 48, 2250 (1993)

36. C. Patrignani and Particle Data Group, Chin. Phys. C 40, 100001 (2016)
37. J. Lahkar, D.K. Choudhury, B.J. Hazarika, Commun. Theor. Phys. 71, 49-55 (2019)

38. N. Isgur, M.B. Wise, Phys. Lett. B 232, 113 (1989)

39. B.J. Hazarika, K.K. Pathak, D.K. Choudhury, arXiv:1012.4377v3

40. A. Deur, S.J. Brodsky, G.F. de Teramond, arXiv:1604.08082v3

41. J. Lahkar, R. Hoque, D.K. Choudhury, Mod. Phys. Lett. A. 34, 1950106 (2019). https://doi.org/10.1142/S0217732319501062

42. B.H. Bransden, C.J. Joachain, Quantum Mechanics, 2nd edn. (Pearson, 2013), p. 363. ISBN:978-81-317-0839-2

43. Heechang Naetal, Phys. Rev. D 86, 034506 (2012). https://doi.org/ 10.1103/PhysRevD.86.034506

44. W. Chen et al., TWQCD Collaboration, Phys. Lett. B 736 (2014). https://doi.org/10.1016/j.physletb.2014.07.025

45. The LHCb Collaboration, arXiv:1304.4741v1 [hep-ex]

46. D. Asner et al., (Heavy Flavor Averaging Group), arXiv:1010.1589

47. B.I. Eisenstein et al., (CLEO Collaboration), Phys. Rev. D 78, 052003 (2008)

48. B. Patel, P.C. Vinodkumar, Chin. Phys. C 34 (2010). arXiv:0908.2212v1 (2009)

49. T. Bames, S. Godfrey, E.S. Swanson, Phys. Rev. D 72, 054026 (2005)

50. B.H. Yazarloo, H. Mehraban, EPL 116, 31004 (2016)

51. F.E. Close, An Introduction to Quarks and Partons (Academic Press, London), p. 396

52. H. Mutuk, Hindawi Adv. High Energy Phys. 2018, Article ID 8095653. https://doi.org/10.1155/2018/8095653

53. J.N. Pandya, P.C. Vinodkumar, Pramana J. Phys. 57(4), 821-827 (2001)

54. R. Van Royen et al., Nuovo Cimento 50, 617 (1967)

55. D.K. Choudhury et al., Pramana J. Phys. 44(6), 519-534 (1995)

56. G.B. Arfken et al., Mathematical Methods for Physics, 7th edn. (2012)

57. D.S. Hwang et al., Phys. Rev. D 53, 4951 (1996)

58. E. Braaten, S. Fleming, Phys. Rev. D 52, 181 (1995). https://doi. org/10.1103/PhysRevD.52.181 